\title{
Pathologic Outcomes during the Learning Curve for Robotic-Assisted Laparoscopic Radical Prostatectomy
}

\author{
Amul Shah, Onisuru T. Okotie, Lee Zhao, Michael R. Pins, Vishal Bhalani, Daniel P. Dalton
}

Department of Urology (AS, OTO, LZ, VB, DPD) and Department of Pathology (MRP), Northwestern University, Feinberg School of Medicine, Chicago, Illinois, USA

\begin{abstract}
Objective: We report our initial experience with 62 patients undergoing robotic-assisted laparoscopic prostatectomy (RALP), focusing on the primary parameter of positive surgical margins. The authors demonstrate that excellent oncologic outcomes can be attained with a less steep learning curve than previously hypothesized.

Materials and Methods: The first 62 patients undergoing RALP by a single physician (DPD) at our institution between November 2005 and August 2007 were retrospectively assessed. Surgical pathology records were reviewed for Gleason score, pathologic tumor stage, nodal status, location of prostate cancer within the specimen, extracapsular extension, surgical margin status, presence of perineural invasion, tumor volume, and weight of the surgical specimen. Margin status was determined using surgical specimens only, and not intraoperative frozen sections. All cases in this series were completed using the four-arm da Vinci Robotic System (Intuitive Surgical, Sunnyvale, California).

Results: Sixty-one patients had prostate cancer on their final surgical pathology specimens. Pathologic stage T2 and stage T3 patients were $88.7 \%$ and $9.7 \%$ of all cases, respectively. The pathologic Gleason score was 7 or greater in $62.3 \%$. Our overall positive surgical margin rate was 3.3\%. Patients with pathologic T2 and T3 disease had a positive surgical margin rate of $1.8 \%$ and $16.7 \%$, respectively.

Conclusions: Our study suggests that RALP can have equal if not better pathologic outcomes compared to open radical prostatectomy even during the initial series of cases. We argue that the learning curve for RALP is shorter than previously thought with respect to oncologic outcomes, and concerns asserting that lack of tactile feedback leads to poor oncologic outcomes are unfounded.
\end{abstract}

Key words: prostate cancer; prostatectomy; laparoscopy; robotic-assisted; outcomes

Int Braz J Urol. 2008; 34: 159-63

\section{INTRODUCTION}

Robotic-assisted laparoscopic prostatectomy (RALP) is becoming increasingly prevalent as the desire for less-invasive procedures continues to grow and the surgery itself becomes more refined. Robotic surgery first appeared in urology in 2000 (1). Since its debut, refinements and the relative increase in ease of the surgery have allowed an increasing number of surgeons to adopt RALP $(1,2)$. Critics have complained of a steep learning curve and lack of tactile feedback during the procedure. Estimates for the number of cases required to complete the learning curve vary widely, ranging from 20 by some authors to 250 by others (3-5). Our study assesses the first 62 RALP surgeries performed by a single physician at our institution, focusing on the primary parameter of positive surgical margins to evaluate oncologic outcomes during the learning curve. 


\section{MATERIAL AND METHODS}

We retrospectively reviewed surgical pathology data for the first 62 consecutive RALPs performed at our institution by a single surgeon (DPD) between November 2005 and August 2007. Although not fellowship-trained in laparoscopy, the surgeon had extensive experience with open radical prostatectomy and laparoscopic renal surgery. Surgical pathology records were reviewed for Gleason score, pathologic tumor stage, nodal status, locations of prostate cancer within the specimen, extracapsular extension, surgical margin status, presence of perineural invasion, tumor volume, and weight of the surgical specimen. One patient had Stage pT0 disease and was excluded from our analysis.

All cases in this series were completed using the four-arm da Vinci Robotic System (Intuitive Surgical, Sunnyvale, California). A transperitoneal approach was used for all cases with 4 robotic arm ports and 2 assistant ports. Pelvic lymphadenectomy was performed if clinically indicated (Gleason score $\geq 7$, PSA $>10$ ). We routinely attempt to spare the bladder neck, as it is our subjective impression this improves early return of continence. Nerve sparing is performed by identifying the plane between the prostatic capsule and neurovascular bundle at the base of the prostate and carrying it distally to the prostatic apex while staying as close to the prostatic capsule as possible. No attempt at a primary lateral release of the neurovascular bundle is made. Posterior dissection consists of dividing Denonvilliers' fascia and dissection along the capsule distally to the prostatic apex. Apical dissection is always performed with apical capsule in view. If a previously placed dorsal vein ligature is obstructing visualization of the apex during dissection, it is removed so the natural planes of dissection are not altered. A second ligature is then placed after apical dissection is complete. A urinary catheter and abdominal drain was placed in all patients. One patient in our series required a blood transfusion.

\section{RESULTS}

Patient and pathologic variables are summarized in Table-1. There was one open conversion for moderate bleeding in a Jehovah's Witness (patient 23 ). This patient had the sole $\mathrm{T} 2$ positive margin. Three additional patients whose procedures were converted (one failure to maintain pneumoperitoneum, two failures to progress) had negative margins. Pathologic stage T2 and stage T3 patients were $88.7 \%$ and $9.7 \%$ of all cases, respectively. The pathologic Gleason score was 7 or greater in $62.3 \%$. Mean prostate weight was $54.4 \mathrm{~g}$ and median tumor volume was $10 \%$. Perineural invasion was present in $68.9 \%$ of patients.

Our overall positive surgical margin rate was $3.3 \%$. Patients with pathologic T2 and T3 disease had a positive surgical margin rate of $1.8 \%$ and $16.7 \%$, respectively. Of the 11 patients in our series who had apical disease, none had a positive surgical margin. Pelvic lymph node dissection (PLND) was completed in $68.9 \%$ of patients. No patient had lymphatic metastasis identified.

\section{COMMENTS}

RALP has the obvious benefit of being minimally invasive; however, in order to become universally adopted, surgeons must be confident that oncologic and functional outcomes are at least equivalent to open surgery. Ahlering et al. concluded that RALP offers the benefits of minimally invasive surgery without compromising clinical or pathologic outcomes (6). Our study confirms that superior pathologic outcomes can be attained even during the initial set of RALP cases performed.

To put into perspective what is an acceptable positive surgical margin rate for RALP, one can refer to the literature for radical retropubic prostatectomy. In a consecutive series of 1,000 cases between 1994 and 2000, Lepor et al. demonstrated a positive surgical margin rate of $19.9 \%$ (7). In a large series of 9,035 cases, Han et al. showed an overall positive margin rate of $14.7 \%(8)$.

Of the 61 RALPs performed and analyzed in our study, only 2 patients $(3.3 \%)$ had positive surgical margins. Current RALP literature has typically demonstrated positive surgical margins between $10-20 \%$. For example, Rozet et al. reported a positive surgical margin rate of $19.5 \%$ (9). Mikhail et al. and Patel et 
Table 1 - Patient and pathology characteristics $(N=62)$.

$\begin{array}{lc}\text { Mean age } \pm \text { SD (yrs.) } & 62.8 \pm 6.8 \\ \text { Pathologic stage (\% of all cases) } & 1(1.6) \\ \text { T0 } & 9(14.5) \\ \text { T2a } & 0 \\ \text { T2b } & 46(74.2) \\ \text { T2c } & 5(8.1) \\ \text { T3a } & 1(1.6) \\ \text { T3b } & 0 \\ \text { T4 } & \\ \text { Postoperative Gleason score } & 23(37.7) \\ 6 & 33(54.1) \\ 7 & 5(8.2) \\ 8 & 0 \\ \text { 9-10 } & 54.4 \pm 17.8 \\ \text { Mean weight (g) } & 10(1-50) \\ \text { Median \% tumor volume (range) } & 68.9 \\ \% \text { Perineural invasion } & 68.9 \\ \% \text { Undergoing PLND } & 0 \\ \% \text { Positive lymph nodes } & \\ \text { Positive margin by location of disease } & 0 / 11 \\ \text { Apical } & 2 / 50 \\ \text { Other } & \\ \text { Number of positive margins by stage } & 1(1.8)^{*} \\ \text { T2 } & 1(16.7) \\ \text { T3 } & 0 \\ \text { T4 } & 2(3.3) \\ \text { All Stages } & \end{array}$

* = patient 23 - Jehovah's Witness who underwent open conversion for moderate bleeding. PLND = pelvic lymph node dissection.

al. reported rates of $16 \%$ and $13 \%$, respectively $(3,10)$. Finally, a large series of 2,652 patients demonstrated a positive surgical margin rate of $13 \%$ (11). When broken down by pathological stage, our data showed positive margin rates of $1.8 \%$ and $16.7 \%$ for stage $\mathrm{T} 2$ and T3 disease, respectively.

Multiple studies show that positive margins following radical prostatectomy are more likely to result from cancers in the apical region than from other regions $(12,13)$. Among our 61 patients, 11 had apical disease, but none had positive margins. In contrast, Mikhail et al. reported positive apical margins in 4 of their 100 patients $(10)$.
A literature review article by Hegarty \& Kaouk showed that positive surgical margin rates were comparable between radical retropubic prostatectomy, laparoscopic radical prostatectomy, and RALP (14). Others have suggested the positive margin rate is related to experience with RALP (15). These studies suggest the margin rate is less a function of the procedure than the experience of the surgeon. It is our belief, however, that the enhanced visualization of important anatomic landmarks provided by RALP results in lower positive margin rates. Anecdotally, it has been the experience of one of the authors (MRP) that prostatectomies by a surgeon who has performed a large number of RALPs generally have intact, non-fragmented capsular tissue making the microscopic assessment of the surgical margins easier.

Our series allows us to draw conclusions that may possibly affect the view regarding usage of RALP in the field of urology. We were able to demonstrate not only that a superior positive surgical margin rate is attainable via RALP, but it is possible without as steep a learning curve as previously hypothesized. To our knowledge, our positive margin rate was lower than any previous RALP series, and we have the added study characteristic that all of the surgeries were done by a single physician. Of course, we recognize that our low positive margin rate would not be possible were it not for the surgeons who pioneered RALP; their work in fine-tuning the surgical technique was essential in attaining our results. Our results indicate that the lack of tactile feedback with RALP has absolutely no impact on oncologic outcomes.

\section{CONCLUSION}

The pathologic outcomes of our study cohort indicate that excellent, perhaps superior, oncologic outcomes can be obtained during the learning curve for RALP. Fear of exposing one's patients to an inferior result should not dissuade the urologist from learning this excellent technique. Furthermore, the lack of tactile feedback with RALP appears to have absolutely no negative effect on surgical margin rates. 


\section{CONFLICT OF INTEREST}

None declared.

\section{REFERENCES}

1. Binder J, Bräutigam R, Jonas D, Bentas W: Robotic surgery in urology: fact or fantasy? BJU Int. 2004; 94 : 1183-7.

2. Schuessler WW, Schulam PG, Clayman RV, Kavoussi LR: Laparoscopic radical prostatectomy: initial shortterm experience. Urology. 1997; 50: 854-7.

3. Patel VR, Tully AS, Holmes R, Lindsay J: Robotic radical prostatectomy in the community setting--the learning curve and beyond: initial 200 cases. J Urol. 2005; 174: 269-72.

4. Herrell SD, Smith JA Jr: Robotic-assisted laparoscopic prostatectomy: what is the learning curve? Urology. 2005; 66 (5 Suppl): 105-7.

5. Ficarra V, Cavalleri S, Novara G, Aragona M, Artibani $\mathrm{W}$ : Evidence from robot-assisted laparoscopic radical prostatectomy: a systematic review. Eur Urol. 2007; 51: 45-55; discussion 56.

6. Ahlering TE, Woo D, Eichel L, Lee DI, Edwards R, Skarecky DW: Robot-assisted versus open radical prostatectomy: a comparison of one surgeon's outcomes. Urology. 2004; 63: 819-22.

7. Lepor H, Nieder AM, Ferrandino MN: Intraoperative and postoperative complications of radical retropubic prostatectomy in a consecutive series of 1,000 cases. J Urol. 2001; 166: 1729-33.
8. Han M, Partin AW, Chan DY, Walsh PC: An evaluation of the decreasing incidence of positive surgical margins in a large retropubic prostatectomy series. $\mathbf{J}$ Urol. 2004; 171: 23-6.

9. Rozet F, Jaffe J, Braud G, Harmon J, Cathelineau X, Barret $\mathrm{E}$, et al.: A direct comparison of robotic assisted versus pure laparoscopic radical prostatectomy: a single institution experience. J Urol. 2007; 178: 478-82.

10. Mikhail AA, Orvieto MA, Billatos ES, Zorn KC, Gong EM, Brendler CB, et al.: Robotic-assisted laparoscopic prostatectomy: first 100 patients with one year of follow-up. Urology. 2006; 68: 1275-9.

11. Menon M, Shrivastava A, Kaul S, Badani KK, Fumo $\mathrm{M}$, Bhandari M, et al.: Vattikuti Institute prostatectomy: contemporary technique and analysis of results. Eur Urol. 2007; 51: 648-57; discussion 657-8.

12. Dahl DM, He W, Lazarus R, McDougal WS, Wu CL: Pathologic outcome of laparoscopic and open radical prostatectomy. Urology. 2006; 68: 1253-6.

13. Ahlering TE, Eichel L, Edwards RA, Lee DI, Skarecky DW: Robotic radical prostatectomy: a technique to reduce pT2 positive margins. Urology. 2004; 64: 1224-8.

14. Hegarty NJ, Kaouk JH: Radical prostatectomy: a comparison of open, laparoscopic and robot-assisted laparoscopic techniques. Can J Urol. 2006; 13 (1 Suppl): 56-61.

15. Atug F, Castle EP, Srivastav SK, Burgess SV, Thomas $\mathrm{R}$, Davis R: Positive surgical margins in robotic-assisted radical prostatectomy: impact of learning curve on oncologic outcomes. Eur Urol. 2006; 49: 866-71; discussion 871-2.

\section{Correspondence address:}

Dr. Daniel P. Dalton

Northwestern Memorial Hospital

201 E. Huron, Suite 10-200

Chicago, IL, 60611, USA

Fax: + 1 312-926-3585

E-mail: daltond12@comcast.net 


\section{EDITORIAL COMMENT}

This initial experience of authors with robotic-assisted laparoscopic radical prostatectomy was very enthusiastic, specially considering that the surgeon had never done pure laparoscopic prostatectomy but had renal laparoscopic skills.

I am very impressive with the very low rate of positive margins in his initial robotic experience, maybe one of the lowest of the literature, even considering surgeons with much more surgeries done. I am intrigued with this and I might consider the possibility that the surgeon had been not so closed to prostatic capsule as he stated. I am curious about the functional results even considering the short follow-up. The space between prostatic capsule and the nerves is very restricted. In order to obtain the best oncologic result one could compromise the functional results and vice-versa. It is well established that successful treatment of prostatic cancer considers three aspects: PSA free, full return of continence and full return of sexual function.

Anyway, this paper encourages those no laparoscopist surgeons to start in robotic assisted laparoscopic prostatectomy.

Dr. Anuar Ibrahim Mitre Division of Urology, General Hospital Sao Paulo University Medical School Sao Paulo, SP, Brazil E-mail:anuar@mitre.com.br 